\title{
An e-Curriculum Based Systematic Resource Integration Approach to Web-Based Education
}

\author{
William W. Song, Anders Forsman, and Jia Yan
}

\begin{abstract}
With the rapid advancement of the web technology, more and more educational resources, including software applications for teaching/learning methods, are available across the web, which enables learners to access the learning materials and use various ways of learning at any time and any place. Researchers from both computer science and education are working together, collaboratively focusing on development of pedagogically enabling technologies which are believed to improve the infrastructure of education systems and processes, including curriculum development models, teaching/learning methods, management of educational resources, systematic organization of communication and dissemination of knowledge and skills required by and adapted to users. In this paper we address the following two aspects of systematic integration architecture of educational systems: 1) learning objects - a semantic description and organization of learning resources using the web service models and methods, and 2) learning services discovery and learning goals match for educational coordination and learning service planning.
\end{abstract}

Index Terms-E-curriculum, learning object, e-learning, web based educational systems.

\section{INTRODUCTION}

For the past decade, the development of technologies for education has been greatly enhanced, from effective organization and management of educational resources such as digital lecturing presentations and course video clips at the early time to flexible and interactive process of learning methods such as self-examination and assessment developed recently. Its ultimate goal is to create a learning environment in which, at any time and any place, learners can access the learning resources, start their learning processes or continue their learning with smooth moving from one stage to another and with supportive self-assessment. With increasing number of educational resources and intelligent teaching and learning techniques available on the Web, we believe that a better learning environment for self-learning and life-long learning will emerge very soon. However, what is the main problem which hinders us to achieve this learning environment? Or how can we build up this learning environment?

According to the education theory, one of the foundational notions - transformation - that is, some kind of change in understanding occurs that transforms cognition from an

Manuscript received April 15, 2014; revised June 15, 2014.

William W. Song and Anders Forsman are with Information Systems, Dalarna University, Borlänge, 78134 Sweden (e-mail: wso@du.se, afm@du.se).

Jia Yan is with Chinese Language Study, Dalarna University, Falun, 791 88 Sweden (e-mail: jya@du.se). initial state to a modified state, and is also somehow observable in behavior, speech, writing, or other forms of production [1], [2]. In other words, a learning process is that of transforming the knowledge developed and embodied in a curriculum by the curriculum designers. The curriculum so developed contains not only the body of knowledge and information that is supposed to be conveyed to the learners, but also the ways of how to do this knowledge transfer (various curriculum models have been developed for this purpose) [3]. Before we propose a curriculum-design based educational system architecture, we consider the three major components in the system and their views.

\section{A. Three Views of e-Learning}

Let us take a look from the angle of the three parties: learners, teachers, and education designers, involving in the process of learning (knowledge transferring). The first angle is from the learners, the main users of an education system. A learner, when intending to learn something, has in mind a purpose, being it for interest or to gain knowledge or a skill. She knows what materials (learning resources) may be suitable for her to start the learning process, for example, something not too difficult. And she may be aware, after a certain period of time of learning, of whether she has satisfactorily gained the knowledge and skills she set to acquire at the beginning. Generally speaking, however, the learners' understanding and awareness of the learning purpose, the level of difficulty of learning resources, as well as the gained knowledge are very initial, general, vague, and even not correct. The second view angle, from the teachers, focuses on the learning process in which the knowledge and skills defined in a curriculum are transferred to the learners. Teachers may be part of the curriculum designers, but most of the time they are implementers of the curriculum development. On one hand, they see clearly what the purposes set for learners on the curriculum and how to assess the learners' outcomes. They can improve the learning effects by providing more flexible ways of teaching and more appropriate teaching materials (but they cannot change the curriculum). On the other hand, they see the problems faced by the learners. They are aware of what approaches should be more suitable to certain students and adaptable to the students with different capabilities and knowledge backgrounds. The third view angle is from the curriculum designers. Usually they are experts in the subjects with rich experiences and knowledge about the overview of the domains, the learning purposes, the ways of learning, and the adoption of the learning materials.

\section{B. Educational System Architecture}


In this section, we brief the importance of (e-)curriculum ${ }^{1}$ design for a systematic collection and organization of educational resources as well as the educational planning, scheduling, and realization. A curriculum is "all the learning which is planned and guided by the school, whether it is carried on in groups or individuals, inside or outside the school" [3]. There are two key features in the definition of curriculum: 1) learning is planned and guided, that is, what goal to achieve and how to achieve it; and 2) it is about schooling, i.e. curriculum is developed (including its theory and practice) within schools and has relation to disciplines, subjects, and lessons (a set of specific knowledge body). From the above definitions we can draw a number of characteristics for curriculum: 1) it contains a body of knowledge in relation to a certain subject, 2) it proposes a methodological and systematical teaching process "methodological" means that a set of teaching and learning methods are developed and adopted and "systematical" means that the content and methods are well connected, linked, and grouped so that a supposedly smooth progress, in terms of features of knowledge to be transferred, should be achieved for learners; 3) in the curriculum development, there is an objective or target for what, after accomplishing the process of teaching and learning (or in other words, realizing the curriculum), to be achieved; and 4) learners are involved in the process of curriculum design, such as learning requirements and outcomes, because everyone has their most suitable way of learning. Learning style inherent in each person is different [4].

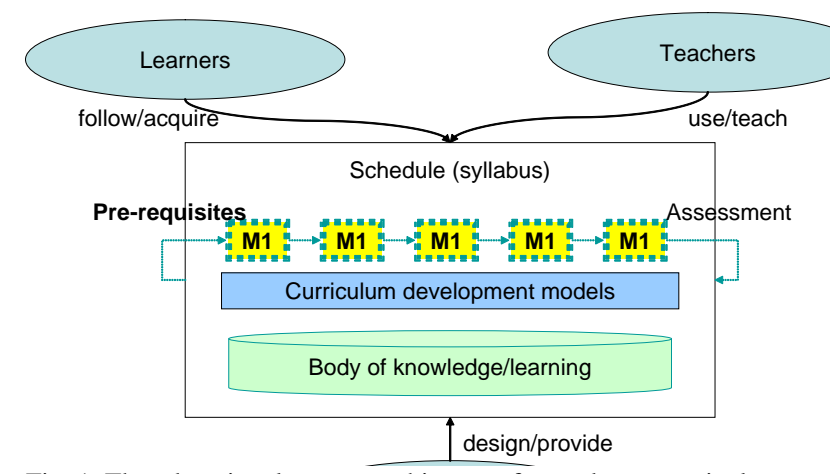

Fig. 1. The educational system architecture focused on e-curriculum.

In this paper, with focusing on the e-curriculum design, we propose a general design architecture of web based educational systems for collecting, analyzing, organizing, scheduling, and building a tailor-made course for learners, intended to meet their learning styles and preferences, see Fig. 1. By a systematic organization of the educational resources from the web, which form a good chunk of subject-related knowledge, educators (curriculum designers) provide a professional support with their knowledge to develop a "personalized" curriculum for a learner using their historic information and learning styles and their previous evaluation results obtained from teachers. In the architecture, it can be

\footnotetext{
${ }^{1}$ We use the term e-curriculum to distinguish it from traditional curriculum design, by considering its use of the web based educational resources, being implemented on the web based media, and being accessed through the web.
}

seen that two layers are applied for organization of the educational e-resources and formation of a learning process, i.e. a sequence of logically assembled modules (self-contained learning blocks). In order to build these two layers of e-curriculum design and tailor-made modules, firstly we take a good look at the current situation of how web based learning resources are organized, which is discussed in next section, secondly we propose the concept of learning object and its definition, as a foundation of organization of learning resources on the web and search mechanism for building "personalized" learning modules, given in Section III, and in Section IV we present a search mechanism based on a learning object (LO for short hereafter) description model including learning pre-requisites, learners' learning styles, and learning goal matching.

\section{E-LEARNING}

Having briefly discussed the general relations among the learners, the teachers, and the curriculum designers, now we can discuss at details these relations in the contexts of online (web based) learning, life-long (flexible styled and on-demand) learning, and self (motivated, organized, and planned) learning. Given the nowadays complex learning environments - massive learning materials available on the web, various learning/teaching methods for selection, and flexible infrastructures such as P2P and Cloud Computing, learners are facing new difficulties and challenges, which can be summarized in the technological views in these five aspects:

1) availability and usefulness of resources,

2) smooth integration of various resources and their presentation,

3) learners' requirements and supposed learning outcomes,

4) automation of learning process in terms of its schedule and interaction, and

5) customization of the resources and agile management of the learning services for delivery as well as necessary human interferences.

The availability of learning resources on the web provides a great convenience for learners to use them at any time anywhere. However, the question is how we can find the learning resources that are available at the time we want them and useful or appropriate to meet our learning objectives. This question is related to: how to describe a learning resource and how to query it. There are a few standards which have been introduced for learning resource description such as IMS ${ }^{2}$ and LOMD [5]. The problem with semantic description for learning resources lies in what granularity, for example a lecture or a course, we will introduce to the description of learning resources, and how formal, such as in a structured form or in a natural language (NL), this description of learning materials can be constructed.

Most of the learning resources, such as online courseware at $\mathrm{MIT}^{3}$, are stored in distributed systems, which are connected to one another via the Internet. They are well

\footnotetext{
${ }^{2}$ http://www.imsproject.org/ and Sharable Content Object Reference Model, http://www.adlnet.gov/scorm/index.cfm

${ }^{3}$ http://ocw.mit.edu/
} 
maintained and can be efficiently queried using Database Systems query languages. For a "standard" use of these learning resources it would not be a problem to have them presented at a best quality. However, in the reality, the learning situations are more complicated. One user may prefer desktop devices while the other handheld devices. One may prefer reading a book while the other watching video shows. Considering people with accessibility difficulties to certain devices, the situations become more complicated. Another question with the learning content presentation is what presentation styles will fit or maximize the use of the learning content and learning methods. Hence, we need to address these two issues: the first is to define, organize and manage learning resources and the second to make the learning resources as services so that we can use the readily developed web service technologies for discovery, composition, and scheduling of learning resources/services. In next section we will define the concept learning objects which form a foundation for all our discussions followed.

How to match a learner's requirements with a collection of learning materials so that the learner will be satisfied with what they learned is always a difficult problem. The first and foremost question is that a learner is not very clear of what she wants to learn since they may have some pre-knowledge about what to learn but not the knowledge (to be learnt) which is a must. The second one is that a learner does not know how to construct her requirements with which the learning systems can satisfactorily find the learning materials. These questions again lead to two design issues: one is how to semantically describe the learning materials - i.e. a usable semantic description framework for e-learning - so that a learner can easily know how to use to describe their needs, and the other is how design architecture can be made for this purpose.

Even though a learner knows her requirements for the purpose of acquiring knowledge, she is most likely unable to construct a schedule or process in which all the required learning materials are organized based on a curriculum for the following reasons:

1) She has not yet understood all the building blocks of knowledge (latter we call them the knowledge points);

2) She has no knowledge (expertise gained from professional training such as education, pedagogy) to organize them into an applicable order (called learning or teaching schedule) for learners;

3) She does not know how to assess what a learner has learnt. Obviously, a key question in the field of online learning, or e-learning, or web based learning is how an e-learning system can automatically (at least semi-automatically) support a potential learner to create a curriculum that best fits her demands. We will address this question in Section IV

\section{LEARNING RESOURCES AND SERVICES}

According to [3], a teaching/learning architecture (structure) consists of these aspects: 1) a learner (student) who wants to learn some knowledge to meet her interest or skills to be more professional, 2) a teacher who uses the curriculum development methods to teach the student systematically so that she can be assessed by the end of the learning process how well she has gained the learning she was set for it, and 3) a curriculum (normalized knowledge and teaching methods) which contains both what students will learn and how to assess the teaching/learning results. The curriculum is the main body of the learning process, which can be considered to be a complex system, see Fig. 1. In this paper, we look at these two tasks of e-learning information system development - constructing learning objects and planning a learning process.

A learning object can be any self-contained and self-described unit in a learning system [5], [6], such as a course, a lecture, or as simple as one book chapter or section. To make the assumption of self-containment and self-description for learning objects aims that when turning them into services, they can be run independently (self-contained) and searched the required (semantic) information in the objects.

\section{A. Learning Objects: A Semantic Description of Learning Resources as Services}

We define a learning object LO as a tuple $<\mathrm{P}, \mathrm{E}, \mathrm{D}, \mathrm{Kp}$, iLO, R>, where

1) $\mathrm{P}$ is a set of pre-conditions that require a learner to posses as pre-requisites of the knowledge before taking LO;

2) $\mathrm{E}$ is a set of effects indicating that after taking LO a learner will have learned or outcomes of a curriculum (if we take a curriculum as a LO);

3) $\mathrm{D}$ is a semantic description of LO, which could a disciplinary ontology [7], indicating that to which topic or subject this LO belongs (when no ontology is available it could be a NL description for the purpose of LO service discovery);

4) $\mathrm{Kp}$ is a subset of the set of knowledge points which have been used in the IEEE/ACM disciplinary hierarchy definitions [7];

5) iLO is a set of sub LOs contained in this LO; if this is empty, this LO is atomic;

6) $\mathrm{R}$ is a set of resources to be consumed by this LO (examples of LO resources can be slides, referencing books or chapters, lecturing audio/video clips, an exercise, or even an experimental working environment).

From this definition, we can see that a LO has these features of connecting LOs. 1) Two LOs are connected (we will term this as a composition of two LO services) through matching their pre-conditions and effects. Of course a composite LO - may contain a number of LOs linked to each other through their pre-conditions and effects to form a super LO. In other words, a LO contains in it a sequence of composite LOs as its sub LOs as in the definition. 2) Each LO in ontology belongs to a parental LO since each LO is associated to a set of $\mathrm{Kp}$ and it is clear that its parental LO contains at least this set of $\mathrm{Kp}$ as its subset. According to this explanation, there is a theoretic semantic hierarchy of LOs a LO ontology, which will be very useful for LO semantic discovery and matches of pre-conditions and effects of LOs.

The introduction of these two special relationships between LOs is essential to (semi-)automation of semantic discovery of a LO, composition of a number of LOs, and a 
goal-effect match. Let us discuss this at details. There are two situations that a LO needs to be searched. The first one when a learner is looking for a learning object to meet her learning demands. Usually a learner does not know what "keywords" in a $\mathrm{Kp}$ set $K P$ specifying a LO and may simply and arbitrarily select a few words $A P$ she thought to be what to be in the LO. Obviously we cannot expect that the two sets KP and AP are the same or even overlapping. However, given that the LO ontology which contains a large number of terms for the LOs, there might be a match, from where starting a reasoning process from a LO which is a parent or an ancestor of the LO searched. A learner can also provide her learning goals (objectives) or even a description of what she wants to achieve by the end of a course, and a goal-effect matching mechanism will help her to find a LO. The second situation is when a LO looks for its preceding LO or a successive LO to make a composite LO. In this case, the LO discovery problem is reduced to a search and match of the pre-conditions of one LO and the effects of the other. The difficulty of LO composition (or that of composite services) mainly lies in which alternatives better meet the learners' need in terms of a variety of quality measurements such as reliability, low cost, availability, accuracy, and so on.

For metadata description of LOs, we can use Dublin Core (DC) [8], a metadata language widely used for library and publication description and management, to describe a LO's $\mathrm{D}$ - description and $\mathrm{R}$ - Learning resources. DC contains fifteen elements, most of which such as title, creator, date, and publication can be flexibly used for describing LOs (as a matter of fact, some of LO standards, such as LOM, are similar to DC). The relations in DC can be used for referencing of a LO to other LOs, which can be further explored to represent more complicated relations between LOs and between a LO to other objects such as learners or LO suppliers. In semantic description, we use OWL-S (a web ontology language for services, recommended by the World Wide Web consortium WWWC) ${ }^{4}$. The key features of OWL-S include a) inputs, outputs, pre-conditions, and effects (IOPE) suitable for describing a LO, b) a hierarchical representation of concepts which can be used for expressing Learning objects in an ontology, and c) an atomic and composite process (LO or service) representation in OWL-S fits well the purpose of atomic and composite LOs. In addition, OWL-S is supported by the first order logic (FOL) which will help the LO system to make well-formed reasoning for LO discovery, composition and scheduling based on the learners' demands.

\section{B. Organization of the Learning Objects (Services)}

A logic structure for a "virtual course" [9] can be briefly illustrated in Fig. 2 as the content of the structure of virtual courses in a server and the process of task decomposition.

A virtual course system, providing various resources about learning materials, can be described as a tree structure, which has three layers in building up it, i.e., server storage convention, application domain standard, and user preference settings. We assume that the server storage convention maintains a similar structure for all the files and

${ }^{4}$ OWL-S, http://www.w3.org/semanticweb/ folders. For each LO, as an item of descriptive information, we need the path from the root (e.g., the server name) to the physical object name.

However, semantic richer information comes from the lower layers, i.e., the application domain standard (e.g., library subject category) and the user settings, where the latter contains more informal semantics. In the reality, we can obtain this kind of ontology from any standard body. For example, an ordinary library subject category (LSC) is this kind of ontology model for learning purpose. The user settings provide more semantic connotations for the concepts of resources but they are quite informal. Currently we manage this part manually. For example, the users need to prepare a resource interdependence graph (RIG) to provide semantic relationships between the resources.

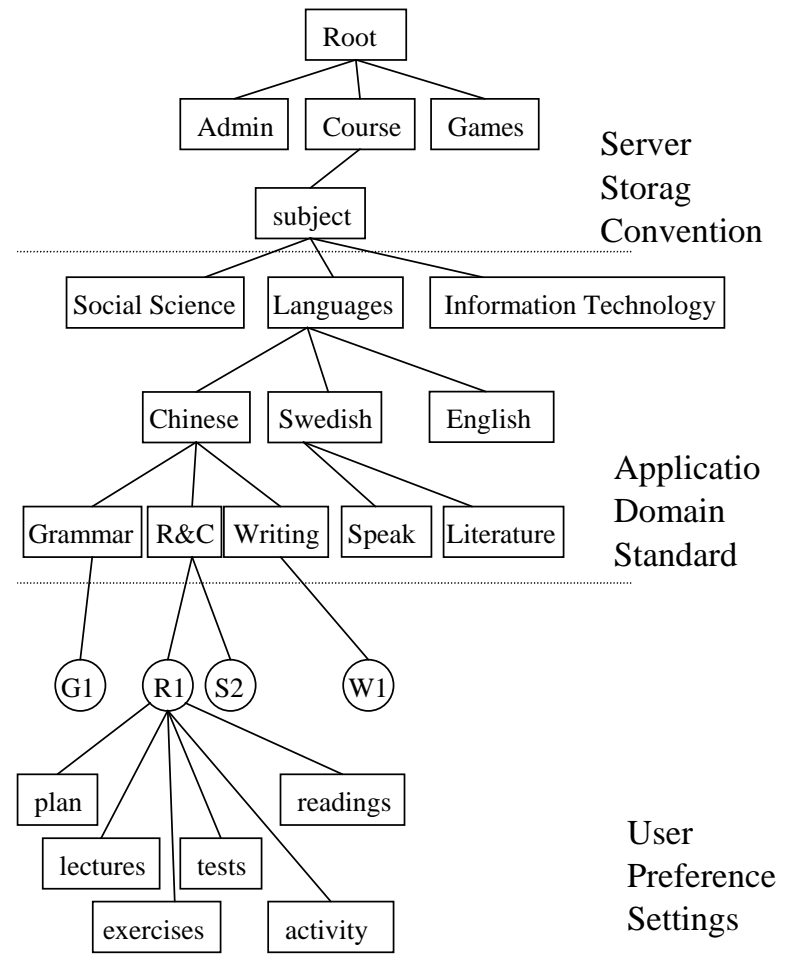

Fig. 2. The example shows the resources in a virtual (web based) course system (VCS) for various courses. Note that G1, R1, S2, and W1 are instances of the courses (conceptual) given above.

\section{Ontology Structure for Learning Objects}

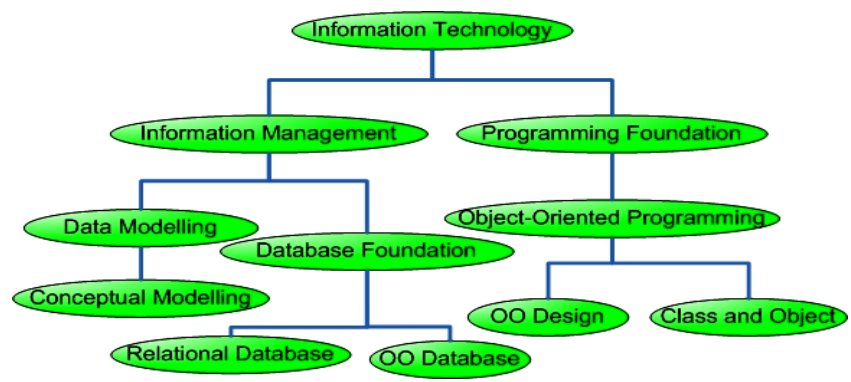

Fig. 3. Part of the learning object ontology for information technology.

The ontology in this example is constructed according to the Computing Curriculum proposed by ACM/IEEE [7], where the knowledge system of information technology is categorized into three levels: Area, Unit, and Topic. The Area represents a sub-domain of the knowledge system that is used to organize, categorize, and describe the top level knowledge 
structure of the knowledge system, such as Information Technology. The Unit represents a sub-direction in the Area, aiming at a more natural organization of the learning components into self-contained groups, such as Database Systems Technologies. The Topic represents a relatively independent content in a Unit and usually constructs one single course, such as DBMS Foundation.

These three levels can make up the ontology of the Information Technology knowledge system, which has been applied to the University Course Online (realcourse in short) system [10] - a Grid based video stream online education service. The leaf nodes of the ontology are the direct parents of LO instances which are normally the titles of the courses or course components that can be freely selected by learners. A LO instance can either represent the whole course or an independent part of the course. Fig. 3 illustrates a part of the ontology.

\section{LEARNING GOAL MATCHING}

\section{A. Learners' Query Structure}

The definition of LOs provides a method of standardizing the representation of learning materials, contents, and applications like experimental methods in teaching/learning processes. Like web services, the learning objects (or services) are provided usually by educational organizations such as schools, universities, and other education oriented communities. It is still difficult and unnecessary for a user to know how a learning object is constructed. We need to make an easy to use query structure, based on the definition of learning objects, for users to express and then match their learning goals. The first important element in such a search goal is what a user wants to find. This element should be expressed explicitly by the user. The element can just be a statement or one word for the subject to search. Since we allow search goals to be unclear, so a goal statement can be in the form of one word or a set of words, stating subjects or topics.

The second element is assigned to be carrier of the learning objects being searched. Of course, we allow "null" as default value for the element. Other than the goal statement and the carrier for searching a learning object, the users' preferences and profiles are also taken into consideration. The preferences and profiles from the learning information consumers are mainly used for tuning the goal matching process. Therefore, a search goal has the following structure:

\{[Goal-statement], [Carrier], [Preference], [Profile]\} where

1) Goal-statement is a list of subjects or topics or a statement of search goal;

2) Carrier is the expected carrier of the learning object to be searched;

3) Preference is a list of statements that the user specifies;

4) Profile is a set of related information on the user.

Now we give some examples to illustrate how a search goal can be matched. Suppose that a university student wants to find some learning materials on Java. He or she may prefer a book on Java for beginners. Then the goal he or she may
Write is:

\{[Programming, Java], [book], [beginner], [university student]\}.

Another example is a search goal from a manager of a marketing department. He hopes to know what is going on with electronic commerce on the market. This goal needs some analysis first before fitting in the search goal structure. The goal needs to be decomposed into a number of sub-goals, e.g. electronic commerce, products, market, economic situation, trends, etc. Since the general goal is vague, the sub-goals are merely made to be better searchable. Therefore, two goals can be defined as follows:

\{[electronic commerce, trend, products], [any], [any], [marketing department, manager]\},

$\{[$ marketing, electronic commerce], [paper], [positive], [-- ]\}.

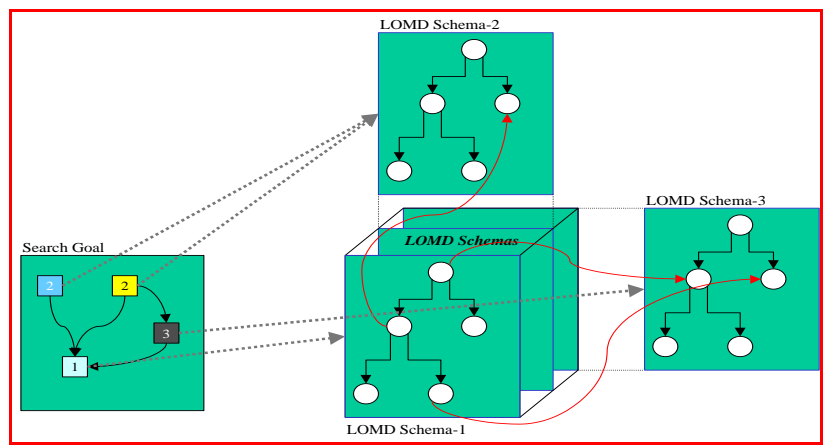

Fig. 4. Goal match: search paths - LODM Schemas are a conceptual representation of e-curriculum structure mapping into concrete learning objects.

\section{B. Search Goal Matching}

After a learning goal is formulated in terms of the LO query structure, starts the search goal match process, dealing with the users' goal structure, e.g., what she or he would like to describe a LO. In general, a user will provide a number of requirements, including search goals and carriers, along with the user's profiles and preferences. The goal match process will compare the user's requirements with the description items (metadata) of the LOs stored and maintained in the repository (remember that the Web is a huge repository for learning objects). Once a goal match is found, either the matched LO will be presented to the user, or further matches continue if there are a number of decomposed goals. Under this circumstance, we call search goal match contains search paths.

Search paths mean that a collection of goal driven requirements, preferences and profiles, is associated to different aspects in the Learning Objects Modelling (LOM) [5], [11] schemas for a learning domain, see Fig. 4. In the figure, the cubic, multi-layered box in the middle is a LOM description of LOs on a learning domain. Each layer is a LOM schema with a hierarchical description of LOs on e.g. a subject or a user preference. Here we show three layers, called respectively LOM Schema-1, LOM Schema-2, and LOM Schema-3. The objects in different layer schemas are connected as e.g. neighbour relationship, displayed by red arrow lines. To the left of the figure, a flat box represents a search goal structure, where search goal statements, carriers, the user preferences, and profiles are included in the 
numbered smaller boxes. Search paths are these dotted arrow lines from the search goal box to the LOM Schemas. This accomplishes the whole process of search goal matching.

The search process is an integrated one since different search elements in the search goal structure are directed toward different LOM schemas first and after goal matches, these search paths are synthesized together to form a meaningful search result. For example, consider this goal structure, \{[marketing, electronic commerce], [paper], [general], [manager]\}. The goal statement contains two sub-goals, marketing and electronic commerce. These sub-goals are analyzed in terms of the LOM Schema- 2 and then the analyzed results will be further checked together with the other results out from the LOM schemas, Schema-1 and Schema-3.

\section{CONCLUSION}

Development of teaching/learning systems and their related curricula has been an everlasting topic. Now as we enter the era of information and knowledge where the Internet and Web provides us a flexible, pervasive, and information-massive platform, it gains a new momentum, that is, learning materials, learning plan, and learning process, as well as the assessment can self be organized by learners in the e-learning environment [12]. In this report, we have tried to address, among others, two problems - describing learning materials (called learning objects here) and matching learning goals, and by defining the concept learning object, present a semantic description model - metadata description, ontology description, and query structure in terms of LOs as a solution to the problems. The proposal of the LOs and related methods has been mainly concerned with a) a formal representation of learning resources to enable (semi-)automation of building learning resources (services) and their management systems for LO discovery and composition and b) a free-styled and natural query formulation to enable learners to use e-learning systems easily.

We have conducted two experiments, a learning objects system development with metadata and ontology modelling and a learning process system development supporting a learner to learn Java programming. In the first experiment, we constructed about two hundreds learning services, including learning resources and learning functions. Using simple keyword-based search and match, the system could find relevant learning objects and make a reasonable sequence of learning process at an acceptable accuracy and performance. Next step for this experiment is to scale up the system to accommodate ten thousands of learning objects (a reasonable number of courses at an ordinary university).

In the second experiment, we focused on building up a learning process, i.e. to match a curriculum with learners' demands and pre-requisites. Learners could input what they wanted to learn, for example, Java Beans and the system would pick up a number of learning objects and organized them into a few learning sequences with all the topics related to Java programming. It was easy that a few book chapters and lecturing presentations were suggested to the learners, and a test with a fixed number of questions (sort of exam) was presented to the learners after they claimed that they finished the book chapters and lecture presentations. It was difficult for the learners to do some Java programming practicals. The learners were presented with a small tool with which they could input their programs and then the system ran them and gave the results as e.g. compilation errors. If a learner tried a few times without successful passing the program compilation, the system should tell her what was wrong and how to correct them. Our next step here is to make this learning process better usable as a curriculum suggests.

In the study of e-learning architecture and systems, we will focus on the solid, theoretical model and architecture that is able to accommodate a variety of learning objects, including people and learning agents, as well as the learning environment. Service science has become an attracting research subject [13], [14] and turning learning objects, including learning contents and learning methods (i.e. actions and behaviours in learning processes) into services, is not only useful for putting LOs in the setting of the Web, but also necessary as applying the service methods and technologies for empowering the applications of LOs and e-learning.

\section{REFERENCES}

[1] N. D. Fleming. (2012). VARK: A guide to learning styles. [Online] Available: www.vark-learn.com/english

[2] C. Haythornwaite and R. Andrews, E-learning Theory and Practice, New York: SAGE Pub. Ltd., p. 272, 2011.

[3] A. V. Kelly, The Curriculum: Theory and Practice, 4th edition, London: Paul Chapman, 1999.

[4] J. Yan and W. Song, "Is it possible to turn educational tools into services?” Journal of Education, vol. 1, pp. 34-39, 2014.

[5] W. W. Song, "A Metadata Framework for Description of Learning Objects," in Proc. the International Conference on Web based Learning, LNCS, Hong Kong, 2002.

[6] X. Du and W. W. Song, "Knowledge based Structure Analysis for Learning Objects Description and Search," in Proc. International Conference on Web based Learning, LNCS, Springer, Edinburgh, Scotland, UK, 2007.

[7] The Writing Committee of CCTT, Computing Curricula, Information Technology Volume, IEEE-CS/ACM, April 2005.

[8] Dublin Core Metadata Element Set. Version 1.1: Reference Description. [Online]. Available: http://dublincore.org/documents/1999/07/02/dces. 1999

[9] W. W. Song and X. Li, "A Conceptual Model for Virtual Organizations in the Grid," presented at the fourth international conference on Grid and Cooperative Computing (GCC05), Beijing, China, Nov. 2005.

[10] J. Zhang and X. Li, "The Model, Architecture and Mechanism behind Realcourse," presented at the IEEE/ACM International Conference on Parallel and Distributed Applications (ISPA'04), Hong Kong, China, Dec. 2004

[11] IEEE Standard for Learning Object Metadata, STD 1484.12.1-2002, 2002.

[12] W. Huang, D. Webster, D. Wood, and T. Ishaya, "An Intelligent Semantic e-learning Framework using Context-aware Semantic Web Technologies," British Journal of Educational Technology, pp. 351-373, vol. 37, no. 3, 2006.

[13] W. W. Song and D. Chen, "An Examination on Service Science: A View from e-Service,"presented at the $17^{\text {th }}$ International Conference on Information Systems Development (ISD’08), Paphos, Cyprus, Aug. 2008.

[14] W. W. Song, Y. Zhong, A. Forsman, X. Zheng, and J. Yan, "Is it possible to build up education software as services?" in Proc. International Conference on Next Generation Learning, Falun, Sweden, 2014, pp. 17-18.

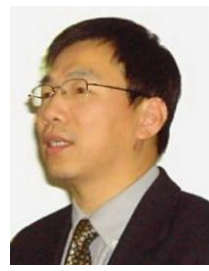

William Wei Song received his BSc in computer science from Zhejiang University, Hangzhou, China in 1982 and $\mathrm{PhD}$ in information systems and sciences from Stockholm University and the Royal Institute of Technology, Stockholm, in Sweden in 1995.

He started his career at a university in 1982. After receiving his $\mathrm{PhD}$ degree, he became a staff researcher at SISU, Sweden from 1995, a senior researcher at ETI, 
Hong Kong University, China from 1999, and an associate professor at Durham University, UK, from 2003. He is now a full professor in business intelligence and information systems at Dalarna University, Sweden. His research interest covers a wide range of fields, including computer science, information systems, artificial intelligence, semantic web, service science, business intelligence, e-business, e-learning, and online education. He has published more than 100 research papers in international journals and conferences.

Professor Song is also a guest professor (researcher) of a number of overseas universities and sits at the board of a number of international journals.

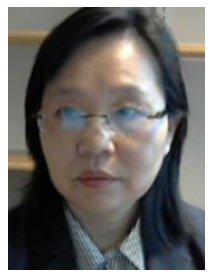

Jia Yan received her MSc in computer science from from Stockholm University and the Royal Institute of Technology, Stockholm, in Sweden in 2001 and B. Ed. From Jinan University, Guangzhou, China.

She is now a lecturer in Chinese language study at Dalarna University, Falun, Sweden. Her research interests include Chinese studies, Chinese literature, and Chinese morphology and etymology. She has been author or co-author of about 10 papers and articles, addressing Chinese language study and online education methods. Mrs. Yan is a member of ORCID.

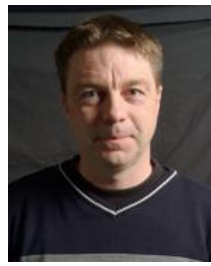

Anders Forsman received his BSc in information system from Luleå University, Luleå, Sweden in 1991 and licentiate degree in information system at Linköping University, Linköping, Sweden in 2005.

$\mathrm{He}$ started his career as a university lecturer from 1993 at Dalarna University and became an assistant head of informatics in 2005 at Dalarna University. In 2009 Forsman became a faculty director of data- and information science at the School of Technology and Business studies, Dalarna University, Sweden.

His research interest covers fields including information system, IT and standards, ITS (intelligent transport systems and services), e-learning and online education. 